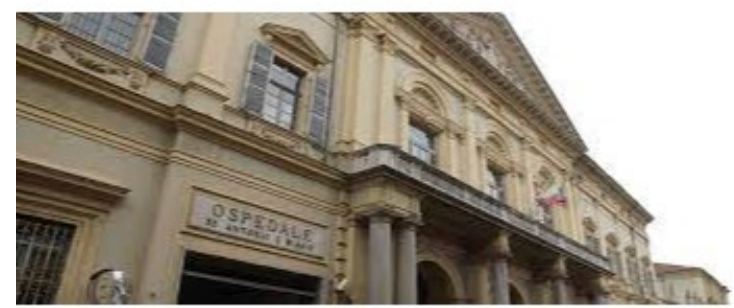

\title{
PREVENTION AND SCREENING OF OSTEONECROSIS OF JAW (ONJ) IN MYELOMA PATIENTS : A MONOISTITUTIONAL FIFTEEN-YEAR EXPERIENCE
}

\begin{abstract}
Giulia Limberti ${ }^{1}$, Antonella Fasciolo ${ }^{1}$, Daniela Tartara ${ }^{1}$, Paola Brigo, Anna Baraldi, Gioacchino Catania ${ }^{1}$, Vittorio Fusco ${ }^{1}$, Elisabetta Ferrero ${ }^{1}$, marco ladetto ${ }^{1}$
\end{abstract}

1 Azienda Ospedaliera SS. Antonio e Biagio e Cesare Arrigo

Funding: The author(s) received no specific funding for this work.

Potential competing interests: The author(s) declared that no potential competing interests exist.

\section{Abstract}

Charts of all myeloma patients observed at the Hematology Unit of Alessandria Hospital (Italy) in years 2005-2020 have been reviewed. Patients received bisphosphonates according to international guidelines, that recommended shorter and tailored duration of antiresorptive treatment (instead of indefinitively prolonged administration, as advised by older guidelines) after 2006-2007. Out of 210 patients receiving pamidronate or zoledronic acid (or sequences of bisphosphonates) and evaluated for oral health, 7 cases of Osteonecrosis of the Jaw (ONJ) were registered (7/210, 3.3\%). However, 5 cases were detected among 41 cases starting antiresorptive treatment before 2006 without preventive visit, and only 2 cases were found among 169 cases receiving dental visit before treatment beginning (2/169, $1.2 \%)$. Our data seem confirm efficacy of measures directed to reduce the risk of $\mathrm{ONJ}$ in myeloma patients treated with less intensive schedules of bisphosphonates, according to latest recommendations..

Background. Bisphosphonates (BPs) are administered in most of myeloma patients. Osteonecrosis of the Jaw (ONJ) is a complication well described in patients treated with IV BPs, such as Pamidronate and Zoledronic Acid (the latter after 2001). The crude frequency rate of ONJ was $6-26 \%$ in some literature series, immediately after the early ONJ reporting (since 2003). Till to 2006-2008, guidelines recommended indefinitely prolonged monthly administrations of IV BPs ${ }^{[1]}$. As a consequence of ONJ appearance and of renal toxicity, following guidelines recommended 1-2 years of treatment and then tailoring on the basis of clinical situation ${ }^{[2][3][4][5]}$. Measures adopted to reduce the ONJ risk (dental visit and dental 
panoramic radiography before start of bisphosphonates; follow-up dental visits during the treatment) have shown a potential reduction of $\mathrm{ONJ}$ rate ${ }^{[6]}$. We investigated whether the occurrence of $\mathrm{ONJ}$ decreased after implementation of preventive measures in patients who received BPs, in a single center experience, updating a previous short-term report ${ }^{[7]}$.

Patients and Methods. We reviewed all patients with Multiple Myeloma treated with BPs between 2005 and 2020. They were observed by a multidisciplinary team (including maxillofacial surgeons, dentists, hematologists, oncologists, nurses, radiologists, nuclear medicine and infective disease specialists). After 2006 patients underwent a baseline oral assessment (dental visit, dental panoramic Rx, eventual tooth avulsion or dental care) before start of treatment. Patients were consequently classified in 3 groups: a) "historical group" (patients starting BP treatment before 2005) b) "screening group" (patients starting BPs without preventive measures, since 2005) c) "prevention group" (patients who started therapy after preventive visit and underwent shorter treatment).

Results At march 2021, 210 patients were included in this analysis. ONJ was observed in 3/21 patients (14.2\%) in group $a$, in $2 / 20$ patients (10\%) in group $b$, and in 2/169 patient from group $c(1.2 \%)$.

Furthermore, 4 other ONJ cases in myeloma patients were referred to the multidisciplinary group by neighboring hospitals (not evaluated by the group before start of treatment).

Conclusions Our data confirmed an important reduction of the ONJ rate in multiple myeloma patients treated with BPs, if preventive measures are applied. Our evaluation with longer follow up seems to confirm that implementation of prevention and reduction of cumulative doses of BPs decreased incidence of ONJ.

\section{References}

1. `James R. Berenson, Bruce E. Hillner, Robert A. Kyle, Ken Anderson, et al. (2002). American Society of Clinical Oncology Clinical Practice Guidelines: The Role of Bisphosphonates in Multiple Myeloma. JCO, vol. 20 (17), 3719 3736. doi:10.1200/jco.2002.06.037.

2. `Martha Q. Lacy, Angela Dispenzieri, Morie A. Gertz, Philip R. Greipp, et al. (2006). Mayo Clinic Consensus Statement for the Use of Bisphosphonates in Multiple Myeloma. Mayo Clinic Proceedings, vol. 81 (8), 1047-1053. doi:10.4065/81.8.1047.

3. ^Brian G.M. Durie. (2007). Use of Bisphosphonates in Multiple Myeloma: IMWG Response to Mayo Clinic Consensus Statement. Mayo Clinic Proceedings, vol. 82 (4), 516-517. doi:10.4065/82.4.516.

4. ^Robert A. Kyle, Gary C. Yee, Mark R. Somerfield, Patrick J. Flynn, et al. (2007). American Society of Clinical Oncology 2007 Clinical Practice Guideline Update on the Role of Bisphosphonates in Multiple Myeloma. JCO, vol. 25 (17), $2464-$ 2472. doi:10.1200/jco.2007.12.1269.

5. ^E. Terpos, O. Sezer, P.I. Croucher, R. García-Sanz, et al. (2009). The use of bisphosphonates in multiple myeloma: recommendations of an expert panel on behalf of the European Myeloma Network. Annals of Oncology, vol. 20 (8), 1303-1317. doi:10.1093/annonc/man796.

6. ^M.A. Dimopoulos, E. Kastritis, C. Bamia, I. Melakopoulos, et al. (2009). Reduction of osteonecrosis of the jaw (ONJ) after implementation of preventive measures in patients with multiple myeloma treated with zoledronic acid. Annals of Oncology, vol. 20 (1), 117-120. doi:10.1093/annonc/mdn554. 
7. ' G. Catania,F. Monaco,A. Baraldi, A. Fasciolo, and A. Pertino. (2014). ONJ (osteonecrosis of jaw) in myeloma patients: a monoistitutional experience of 117 patients treated with bisphosphonates. Ann Stomatol (Roma). 2014 Apr-Jun; 5(2 Suppl): $7-8$. 